\title{
COME AFFRONTARE LO STUDIO DELLA GRAMMATICA LATINA ALLA LUCE DELLA LINGUISTICA CONTEMPORANEA
}

\section{RENATO ONIGA}

Dipartimento di Lingue e Letterature, Comunicazione, Formazione e Società Università degli Studi di Udine (Italia)

renato.oniga@uniud.it

Recibido: 11/05/20

Aceptado: 15/08/20

The development of General Linguistics in recent decades has produced important innovations in the knowledge of the functioning mechanisms of ancient and modern languages. However, these innovations have not yet fully entered the study of the Latin language. In this contribution, examples of application of the so-called "new comparative method" to some traditional problems of Latin grammar are proposed. In particular, the paper presents a description of the structure of the syllable and of the Latin accent law, a proposal for analyzing the structure of morphemes, and an introduction to the phrase structure.

\section{Latin / Grammar / Linguistics / Teaching / Philology}

\section{CÓMO ABORDAR EL ESTUDIO DE LA GRAMÁTICA LATINA A LA LUZ DE LA LINGÜÍSTICA CONTEMPORÁNEA}

El desarrollo de la Lingüística general en las últimas décadas ha producido importantes innovaciones en el conocimiento de los mecanismos de funcionamiento de las lenguas antiguas y modernas. Sin embargo, estas innovaciones aún no han entrado completamente en el estudio de la lengua latina. En esta contribución, se proponen ejemplos de aplicación del llamado método neocomparativo a algunos problemas tradicionales de la gramática latina. En particular, el artículo presenta una descripción de la estructura de la sílaba y de la ley del acento latino, una propuesta para analizar la estructura de los morfemas y una introducción a la estructura de los sintagmas.

\section{Latín / Gramática / Lingüística / Enseñanza / Filología}




\section{Introduzione}

Negli ultimi decenni, la linguistica generale ha compiuto notevoli progressi nella conoscenza di alcuni meccanismi fondamentali che stanno alla base del funzionamento della grammatica delle lingue. Anche lo studio del latino potrebbe utilmente confrontarsi con queste innovazioni, per proporre agli studenti un'esposizione della materia grammaticale più adeguata al mondo di oggi. Purtroppo però questo confronto non è ancora entrato nella pratica dell'insegnamento: la generalità dei manuali di grammatica latina oggi in uso nelle scuole del mondo rispecchia lo stato delle conoscenze nella linguistica generale raggiunto tra la seconda metà dell'Ottocento alla prima metà del Novecento. In seguito, il rapporto tra latino e linguistica si è interrotto: il ruolo delle lingue classiche nel pensiero dei principali linguisti del XX secolo, da Ferdinand de Saussure a Noam Chomsky, è assolutamente marginale, e viceversa, la linguistica moderna, dallo strutturalismo alla grammatica generativa, è rimasta assolutamente marginale per $\mathrm{i}$ filologi e ancor più per gli insegnanti di lingue classiche.

Privato della sua relazione privilegiata con la linguistica, l'insegnamento del latino è dunque oggi in difficoltà: gli insegnanti sono spesso demotivati e gli studenti falliscono. Si tratta di un problema che riguarda la didattica specifica della disciplina, non la didattica generale. Il disagio del latino non deriva dall'incapacità di passare dal sapere al saper insegnare: il problema sta proprio in un sapere che è entrato in crisi. Non basta mettere su internet un corso tradizionale per renderlo miracolosamente moderno ${ }^{1}$. Il punto cruciale è il valore scientifico della materia grammaticale. Lo studio del latino ha compiuto dei progressi quando ha saputo confrontarsi con la linguistica e rinnovarsi. Per questa ragione, il programma educativo basato sulla lettura dei testi classici con commento grammaticale è stato a lungo ritenuto utile per il suo valore pedagogico generale. Oggi tutto ciò viene messo in discussione.

\section{I metodi oggi in uso per lo studio del latino}

Schematizzando, vi sono oggi essenzialmente tre metodi per la didattica del latino: a) Grammatica tradizionale; b) Storia e cultura; c) Metodi diretti ${ }^{2}$.

Il primo metodo si basa sulla grammatica tradizionale, che presenta la materia come uno strumento di carattere pratico, secondo una vulgata che parte dai grammatici tardo-antichi e si ferma alla linguistica del XIX secolo. L'anacronismo non è neppure percepito, oppure è orgogliosamente affermato come antidoto alla modernità. Il dialogo tra grammatica e linguistica è semplicemente rifiutato, come se fossero due discipline diverse, senza punti di contatto. Tutto ciò che è stato prodotto dalla linguistica negli ultimi decenni è visto con sospetto, e considerato inutile, se non dannoso.

Questo metodo ha l'evidente difetto di essere ormai obsoleto, impermeabile ad ogni rinnovamento, rinchiuso in una dimensione dogmatica e acritica che contrasta 
con i più elementari requisiti del metodo scientifico. Così si perde inoltre il secolare ruolo della grammatica latina come modello per le lingue moderne: il latino e il greco non sono mai stati un sapere di nicchia, riservato solo ai futuri classicisti, ma sono stati essenziali per la formazione linguistica di base dell'intera élite intellettuale: medici, avvocati, scienziati, che non possono certamente rinunciare al confronto con la modernità .

Pertanto, negli ultimi decenni, si è diffuso un secondo metodo, che ha progressivamente trasformato il latino da disciplina linguistica a disciplina storicoculturale. In Italia, la materia scolastica che una volta era chiamata "lingua latina" è ora divenuta nella terminologia ufficiale "lingua e cultura latina", con l'enfasi che si sposta sempre più dalla lingua alla cultura, dalla forma ai contenuti. Al centro è posto il significato generale della civiltà antica, valorizzando la storia, l'archeologia, la filosofia, e più recentemente l'antropologia culturale ${ }^{4}$. Anziché la letteratura, si privilegia lo studio della vita quotidiana. Questa tendenza è particolarmente diffusa e apprezzata in Germania e in Inghilterra, dove negli ultimi decenni lo strumento didattico più utilizzato per l'insegnamento del latino è il Cambridge Latin Course (1966), che utilizza molte illustrazioni, in particolare i fumetti, ma anche foto di oggetti antichi e siti archeologici ed è dotato di una versione interattiva su internet ${ }^{5}$.

In questo metodo vi sono certamente aspetti positivi. Un approccio multimediale all'insegnamento è senza dubbio adatto alla nuova generazione dei cosiddetti nativi digitali. Gli studenti educati secondo questo metodo saranno sicuramente in grado di visitare con competenza un museo archeologico, riconoscere le analogie e le differenze tra il mondo antico e quello moderno, evitare qualsiasi abuso della retorica nell'idealizzare il mondo classico come un modello astratto e astorico.

Ma ci sono anche aspetti negativi. In primo luogo, il linguaggio non è solo un prodotto della cultura, ma anche della natura ${ }^{6}$. A partire da Saussure sappiamo che la lingua è un sistema che funziona in un certo modo per necessità interna, a prescindere dalla storia e dalla cultura. Come poi è sostenuto da CHOMSKY, il linguaggio non è stato "inventato" dall'uomo, ma si è formato non diversamente da altre caratteristiche del nostro sistema cognitivo, come la vista o l'udito. Perciò, è necessario studiare il linguaggio non solo come un fenomeno culturale, ma anche come un fenomeno naturale. Questo aspetto, che già era implicito nella vecchia tradizione grammaticale, non può essere abbandonato, pena la perdita di comprensione di ciò che propriamente è la natura del linguaggio.

In secondo luogo, esiste il rischio di concentrarsi troppo sugli aspetti della vita quotidiana e perdere di vista quella che da sempre è chiamata la "cultura umanistica". Essa si esprime non tanto nella varietà degli usi e costumi, ma nei capolavori della letteratura, i "classici" di valore universale. Se si perde la capacità di capire nella lingua originale il valore artistico di capolavori espressivi della letteratura mondiale, e si riduce lo studio del mondo antico all'esplorare una cultura folclorica simile a tante altre, bisognerebbe chiedersi perché lo studio delle culture europee antiche dovrebbe avere ancora un ruolo privilegiato rispetto alle innumerevoli 
culture, presenti e passate, dell'est e del sud del mondo, che sono sempre più presenti nell'universo globalizzato.

Arriviamo così al terzo metodo, secondo il quale al contrario la lingua prevale sulla cultura, in modo tale da proporre lo studio del latino a partire da materiali linguistici semplificati, senza la mediazione dell'analisi grammaticale, ma applicando le stesse metodologie audio-orali utilizzate nell'insegnamento delle lingue moderne. Questo metodo si auto-definisce perciò "diretto", "naturale" o "natura" (MOST 1958; ØRBERG 1954). In Italia si è diffusa in particolare una variante che fa riferimento al manuale di ØRBERG, basato sulla lettura intensiva di testi latini artificiali molto semplici, finalizzati ad attivare le competenze linguistiche nella lettura, nella scrittura e nel discorso (MIRAGLIA 2004).

L'aspetto positivo di questo metodo è di affermare che il latino, prima di diventare una lingua letteraria codificata nei testi classici, è stato una lingua naturale come altre. Possiamo così riscoprire una dimensione del latino come lingua viva, che ha dominato a lungo in Europa dal Medioevo fino all'inizio del XIX secolo, quando lo studio del latino era finalizzato non solo alla lettura dei testi classici, ma anche e soprattutto all'acquisizione della capacità di parlare e scrivere in latino, perché questa lingua era usata come mezzo di comunicazione internazionale in varie aree centrali della vita sociale, dalla medicina alle scienze naturali, dalla legge alla religione.

Gli aspetti problematici sono però molti. In primo luogo, questo metodo si basa sul sostanziale equivoco tra acquisizione, che riguarda i bambini, e apprendimento, che riguarda adolescenti e adulti. Dato che oggi lo studio del latino si sta spostando verso età decisamente più avanzate rispetto al passato, un metodo fondato sull'acquisizione spontanea, più che sull'apprendimento razionale, va nella direzione sbagliata. Inoltre, la lingua della comunicazione internazionale non è più il latino, ma l'inglese: quindi, parlare e scrivere in latino non è un'abilità che offra particolari vantaggi nella vita di tutti i giorni o nel mondo delle professioni. Ma soprattutto, questo metodo fallisce quando si passa dalle storielle scritte in una lingua artificiale alla lettura dei classici veri. Ciò accade perché è sbagliato applicare metodi concepiti per l'apprendimento di lingue vive ad una lingua che non ha più parlanti di madrelingua, ma solo testi letterari. Il metodo di ØRBERG è caratterizzato dall'uso di testi artificiali, limitati nei loro contenuti culturali e insignificanti a livello letterario. $\mathrm{Ci}$ si può divertire imparando a parlare questo tipo di pidgin, che rinnova i fasti di ciò che gli umanisti chiamavano latino "barbaro", se non "maccheronico". Ma tutto ciò non agevola il necessario passo successivo nello studio del latino, ovvero la lettura di testi classici autentici, che sono altamente strutturati e complessi, come tutti i capolavori della letteratura universale, per capire i quali non basta essere parlanti di madrelingua. Infine, questo metodo va nella direzione opposta, rispetto alle raccomandazioni delle scienze dell'educazione, che negli ultimi decenni sono passate dal paradigma comportamentista a quello cognitivista. Per ottenere buoni risultati pratici, in qualunque disciplina, è ormai evidente che non serve l'addestramento basato sulla ripetizione acritica, ma occorrono piuttosto 
l'apprendistato cognitivo e l'educazione intellettuale. Bisogna cioè apprendere razionalmente: imparare a pensare, più che agire in modo inconscio e automatico (COLLINS et al.1995; BALDACCI 2012: 315-320).

\section{Una nuova prospettiva: il metodo neo-comparativo}

Per cercare di uscire da questa impasse, che riguarda certamente l'insegnamento delle lingue classiche, ma anche quello delle lingue moderne, negli ultimi decenni è stato sviluppato un nuovo metodo, chiamato "neo-comparativo"7. Rispetto al comparativismo classico della linguistica indoeuropea, il nuovo metodo compara le lingue per ricostruire non la loro storia, ma la loro struttura, sulla base della cosiddetta Grammatica Universale, cioè l'insieme di costanti e variabili su cui è costruita ogni lingua. Questo metodo mira perciò a promuovere il confronto tra il latino e le altre lingue antiche e moderne che lo studente si trova ad affrontare nel proprio percorso di studi.

Tutto ciò presuppone un dialogo critico e nello stesso tempo costruttivo tra la grammatica tradizionale e la linguistica contemporanea ${ }^{8}$. Ogni aspetto della grammatica dovrebbe essere conquistato dallo studente come una scoperta, secondo un metodo scientifico, che parte dall'osservazione dei dati, individua problemi interessanti e procede all'elaborazione di teorie in grado di risolverli ${ }^{9}$.

L'obiettivo qualificante dello studio del latino nella scuola non dovrebbe essere la formazione di filologi classici o parlanti e scrittori latini, ma lo sviluppo di una cultura linguistica aperta e inclusiva per tutti ${ }^{10}$. In questa prospettiva, si può difendere il ruolo del latino nel percorso formativo scolastico di base a livello globale, sia nel curriculum scientifico sia in quello umanistico, con ricadute positive sulla conoscenza consapevole della propria madrelingua e delle lingue straniere.

La grammatica latina studiata con questo metodo può tornare insomma ad essere una disciplina di attualità, non però per ragioni esterne, cioè l'uso di tecnologie o metodi didattici generali, ma per un rinnovamento dall'interno. Non è necessario cambiare tutto: la grammatica tradizionale, accanto a errori e anacronismi, contiene concetti utili: è una ricchezza da non dilapidare. Contrariamente a certe illusioni moderniste, innovare non significa fare tabula rasa del passato. Tanto meno si può indulgere a facili scorciatoie o illusioni di metodi miracolosi che permettano di imparare tutto e subito senza sforzo, magari con l'esibizione di effetti speciali tecnologici.

Gli insegnanti che conoscono bene la grammatica tradizionale non si dovrebbero trovare a disagio nel confronto con la linguistica di oggi. Il gusto del filologo classico per la descrizione completa e dettagliata dei fenomeni è anche quello del linguista. La linguistica contemporanea non è poi così lontana dalle speculazioni dei grammatici antichi. Si tratta spesso di affrontare gli stessi problemi, che hanno le loro radici nell'antichità classica. L'idea di una grammatica "generale e ragionata" si trova già a Port-Royal e l'affermazione di Anatole France che il latino "insegna a ragionare" contiene una profonda verità. Ma per poterla riproporre oggi 
senza anacronismi, la si deve ripensare alla luce della linguistica e della pedagogia contemporanea. Si tratta di passare dalla concezione della grammatica come un insieme di norme preconcette all'elaborazione della stessa come uno strumento razionale, che cerca di spiegare in modo scientifico il funzionamento di una lingua naturale. L'impostazione nozionistica della grammatica tradizionale deve dunque essere sostituita da una presentazione critica per problemi.

Ricordiamo che Noam CHOMSKY ha spesso sottolineato la propria ispirazione al metodo educativo del filosofo John DEWEY ${ }^{11}$. Proprio a partire dalla teoria del "pensiero riflessivo" di Dewey, la pedagogia degli ultimi decenni, con l'affermarsi delle nuove prospettive di tipo problematicista e cognitivista, ha superato il vecchio spontaneismo che aveva messo in crisi l'insegnamento tradizionale del latino, e ha rivalutato il contributo delle didattiche disciplinari alla formazione della mente e della personalità ${ }^{12}$. Si potrà in tal modo difendere l'idea tradizionale che lo studio del latino forma la mente, ottenendo benefici educativi generali, quali il risveglio della curiosità intellettuale e dell'interesse verso la materia, nonché lo sviluppo della capacità di ragionamento. Nei paragrafi seguenti, vedremo come è possibile rinnovare secondo questo metodo la presentazione di alcuni punti fondamentali per lo studio della grammatica latina ${ }^{13}$.

\section{Problemi di fonetica}

Secondo la tradizione dei grammatici latini, che iniziavano la loro esposizione trattando de littera e de syllaba, ancor oggi i manuali iniziano la descrizione del latino con un capitolo dedicato alla fonetica, generalmente trattato in maniera molto rapida e sommaria nella pratica comune dell'insegnamento. Si tratta invece di un settore molto interessante, nel quale la linguistica contemporanea ha compiuto grandi progressi.

In primo luogo, vale la pena di valorizzare una caratteristica fondamentale del linguaggio umano: la capacità di combinare insieme unità più piccole per formare unità più grandi. Questo lo sapevano già gli antichi, ma il punto fondamentale scoperto dalla linguistica di oggi è che questa combinazione non avviene per addizione lineare, ma le unità sono organizzate in una struttura, caratterizzata da una gerarchia d'inclusione.

Il principio di dipendenza dalla struttura è una delle proprietà fondamentali della Grammatica Universale. Dati tre elementi linguistici, X, Y e Z, la computazione del linguaggio umano non considera pertinente solo la loro successione lineare XYZ, ma anche il loro raggruppamento. Dunque ci sono due possibilità: la prima è che $\mathrm{X}$ e Y si uniscano per formare un'unità, a cui poi si aggiunge $Z$; la seconda è che invece siano Y e Z a formare un'unità, a cui poi si aggiunge X. Questa proprietà fondamentale sta alla base di tutta la grammatica, dalla fonetica alla morfologia e alla sintassi.

Per quanto riguarda la fonetica, oggi sappiamo che la struttura della sillaba è formata da tre elementi: l'attacco, il nucleo e la coda. Il punto essenziale è che il 
nucleo assieme alla coda forma la rima. La struttura gerarchica della sillaba è rappresentata dal seguente diagramma ad albero, che mette in evidenza la gerarchia della struttura, ad esempio nella sillaba cum-:

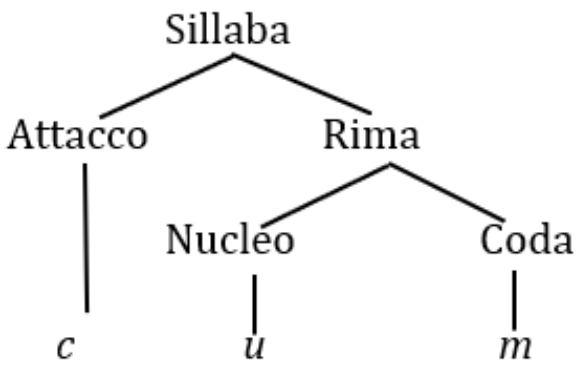

La struttura della rima ci permette di definire che una sillaba aperta ha una rima senza coda, mentre una sillaba chiusa ha una rima dotata di coda. Questa definizione è più precisa di quella tradizionale, che definisce sillaba aperta quella che finisce in vocale, e sillaba chiusa quella che finisce in consonante. Ma vi sono anche sillabe che finiscono in dittongo, che propriamente non è né una vocale né una consonante. Il secondo elemento di un dittongo è una semivocale in posizione di coda, dunque queste sillabe sono chiuse.

La distinzione tra sillabe aperte e sillabe chiuse è fondamentale per capire il concetto di quantità sillabica. Ogni vocale è breve o lunga per sua natura, ma la quantità sillabica dipende appunto dalla struttura della sillaba. Precisamente, è breve una sillaba aperta con vocale breve (ad es. $p \breve{a}$-), mentre tutte le altre sillabe sono lunghe (ad es. $p a \breve{l}-, p \bar{a}-, p \bar{a} c-$, pae-). La spiegazione tradizionale fa riferimento alla durata dei fonemi: "La durata è massima nella vocale, minore nella consonante di chiusura, minima -anzi istantanea- nella consonante d'inizio. Nell'economia fonologica dei Latini erano percepite e apprezzate dall'orecchio le durate della vocale e della consonante di chiusura, non quella della consonante d'inizio" (TRAINA $\mathcal{E}$ BERNARDI PERINI 1992: 84).

Ma questa spiegazione non è valida. Anche se la durata delle consonanti è minore in apertura che in chiusura, questa non può diventare istantanea. Una sillaba come stra- dura sicuramente più di una sillaba come ac-. Dunque, ciò che conta non è la durata, ma la struttura della rima, se sia o no dotata di coda. Si tratta di un fenomeno fonetico universale, che vale per tutte le lingue, non solo per il latino, e si basa sul fatto che la sequenza dei fonemi nella sillaba non è lineare, ma nucleo e coda formano tra di loro un'unità, che è la rima. Soltanto la struttura della rima determina la lunghezza della sillaba.

Questa prospettiva ci permette inoltre di riformulare la legge fondamentale dell'accento latino. Anche in questo caso, l'approccio della grammatica tradizionale ha dei limiti, sia di carattere descrittivo (è troppo complessa), sia di carattere esplicativo (non si basa su proprietà universali del linguaggio). Secondo la tradizione grammaticale vi sarebbero ben tre leggi dell'accento latino, cioè: 
(a) Legge del trisillabismo: "l'accento non può risalire oltre la terzultima".

(b) Legge della baritonesi: "l'ultima sillaba non è accentata".

(c) Legge della penultima: "penultima lunga porta l'accento, penultima breve fa accentare la terzultima”.

In realtà, tutta questa complicazione non è necessaria. Più semplicemente, si può determinare la posizione dell'accento latino con un unico algoritmo fonologico: "esclusa l'ultima sillaba, si risale verso l'inizio della parola: l'accento cade sulla prima sillaba lunga o il primo elemento di un gruppo di due sillabe brevi che incontriamo" ${ }^{14}$.

Dunque, l'accento cade sulla penultima sillaba se è lunga (patrốnus): si tratta ovviamente della quantità sillabica, e non della quantità vocalica, ad esempio la parola sepultus si accenta sepúltus, perché la sillaba è lunga (pul-), anche se la vocale è breve $(\breve{u})$. Quando invece la penultima sillaba è breve, l'accento cade sulla terzultima, che sarà necessariamente una sillaba lunga (trếdecim), oppure la prima sillaba di una coppia di brevi (fắcĕre).

Questa presentazione ha il pregio dell'economia descrittiva, dal momento che propone una sola regola contro tre. Inoltre, ci permette di spiegare il significato della regola all'interno nel sistema fonologico. L'accento cade sempre davanti all'ultima sillaba, su un elemento fonologico che è formato da una sillaba lunga o da una coppia di sillabe brevi con accento sulla prima: tale elemento è chiamato dalla linguistica "trocheo moraico" (MESTER 1994).

Un trocheo moraico è un piede fonologico formato da due more, delle quali la prima porta l'accento. La "mora" è la misura di una sillaba breve, mentre una sillaba lunga ha la misura di due more. Il trocheo moraico può essere realizzato pertanto da un gruppo di due sillabe brevi con accento sulla prima (sắp ̌s) o da una sillaba lunga $(t \dot{u})$, e naturalmente quando la sillaba lunga è realizzata da un dittongo, l'accento cade sulla prima mora (áut).

La necessità di formare un trocheo moraico che porti l'accento spiega inoltre perché i monosillabi devono essere sempre lunghi (dó, dát $)^{15}$, mentre i bisillabi possono avere una penultima lunga (báca), oppure una coppia di brevi (tŏgă). Possiamo inoltre capire il problema dei bisillabi giambici (ĕgō), che essendo

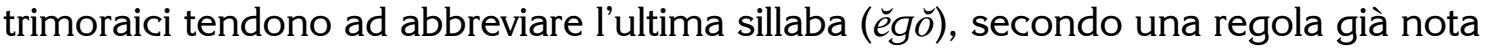
alla tradizione, detta correptio iambica "abbreviamento giambico".

Oltre all'economia descrittiva, questa analisi ha il pregio di una maggiore adeguatezza esplicativa. Credo sia importante per lo studente capire che l'accento latino non segue leggi bizzarre, ma una norma di fonetica accentuale che è tipica di molte lingue naturali.

La regola dell'accento latino è infatti molto simile a quella dell'accento inglese nel caso di nomi formati da un solo morfema (CHOMSKY \& HALLE 1968: 71). Esclusa l'ultima sillaba, l'accento cade sulla penultima se contiene una vocale lunga (hiátus, Minnesóta) oppure se è una sillaba chiusa (amálgam, uténsil), altrimenti risale sulla terzultima, analogamente a quanto accade in latino (jávelin, América). 
Una regola simile si ritrova anche in italiano: se escludiamo come eccezionali le parole tronche (città) e bisdrucciole (pòrtamelo), l'alternanza dell'accento tra le parole piane e sdrucciole è regolata proprio dal medesimo principio del trocheo moraico: l'accento cade sulla penultima sillaba se è lunga, oppure sulla coppia di brevi davanti all'ultima sillaba (subìto/sùbito).

\section{Problemi di morfologia}

Passiamo a qualche riflessione sui meccanismi fondamentali della morfologia. Partiamo ancora dal principio della Grammatica Universale che determina il raggruppamento di unità più piccole per formare unità più grandi. Si tratta di fatto già noto agli antichi: una tipica ars grammatica procedeva infatti dal semplice al complesso, trattando in sequenza le lettere, le sillabe, le parole e le frasi, come leggiamo ad esempio in Prisciano:

Quemadmodum litterae apte coeuntes faciunt syllabas, et syllabae dictiones, sic et dictiones orationes. (Prisc. G.L. 3.108, 9-10).

Come le lettere opportunamente combinate formano le sillabe, e le sillabe le parole, così le parole compongono le frasi.

Rispetto a questa formulazione, però la linguistica contemporanea ci permette di aggiungere una cosa importante. Una parola può essere scomposta non solo in lettere e sillabe, che di per sé non sono dotate di significato, ma anche nei suoi elementi dotati di significato, che sono chiamati morfemi. Ad esempio, la parola inglese books dal punto di vista fonetico è formata da una sola sillaba, ma dal punto di vista morfologico è formata da un morfema lessicale book e un morfema grammaticale $-s$. Similmente, la parola italiana libri dal punto di vista fonetico è composta dalle sillabe li-bri, ma dal punto di vista morfologico è composta dai morfemi libr-i.

Dunque la morfologia è un sistema indipendente dalla fonetica, e si basa appunto sulla struttura dei morfemi. Questo fenomeno era ignoto agli antichi, che non conoscevano quella che oggi chiamiamo la "doppia articolazione" del linguaggio (fonetica e morfologica), ma sapevano scomporre la parola solo dal punto di vista fonetico in sillabe e lettere, mentre dal punto di vista morfologico la parola, in quanto parte del discorso, era un tutt'uno non analizzabile:

Verbum dico orationis partem quae sit indivisa et minima (Var. L. 10.77).

Chiamo parola la parte del discorso che sia la più piccola e indivisibile 
Questa affermazione non è in contraddizione con la precedente di Prisciano: gli antichi conoscevano solo la prima articolazione del linguaggio, quindi la parola era divisibile dal punto di vista fonetico ma indivisibile dal punto di vista morfologico. Per questo motivo, nella pratica scolastica è rimasto il procedimento di imparare filastrocche di parole non analizzate in morfemi: rosa, rosae, rosae; laudo, laudas, laudat, ecc., che risalgono ai tempi antichi in cui la parola era appunto un tutt'uno non analizzabile.

Solo a partire dall'Ottocento, grazie all'influsso dei grammatici indiani, sono stati scoperti i concetti di radice, tema e desinenza, che ormai sono entrati anche nella tradizione grammaticale. Ciò che ancora non è sufficientemente noto, è che anche per i morfemi, come per i fonemi, esiste non solo una sequenza lineare, ma anche una struttura gerarchica, che può essere schematizzata dal seguente diagramma ad albero per la parola amabamus (Cfr. EMBICK 2015):

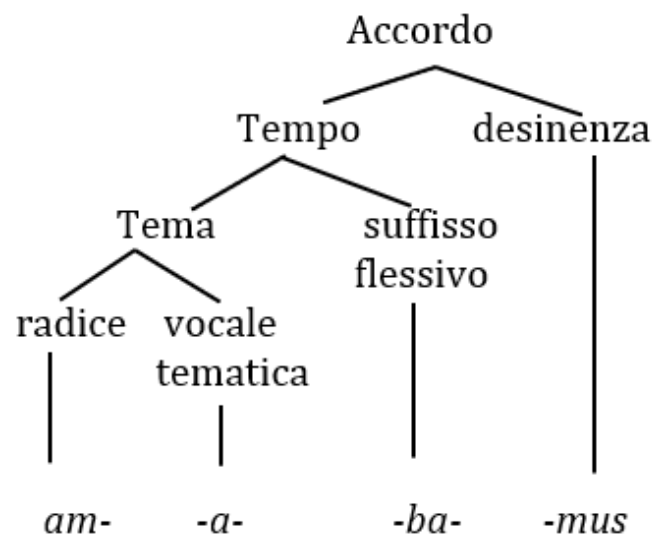

La radice più la vocale tematica forma il Tema, che esprime l'appartenenza della parola ad una parte del discorso, in questo caso un verbo. Il Tema più il suffisso flessivo forma poi il tema temporale, che esprime appunto il Tempo (nell'esempio, l'imperfetto), e infine la desinenza aggiunge le informazioni di persona e numero, essenziali per l'accordo tra verbo e soggetto (nell'esempio, prima persona plurale).

Dunque, un suggerimento per la didattica della morfologia, è di porre il problema di come funziona il meccanismo della flessione in sé, al di là delle singole forme da imparare a memoria. Si dovrebbe focalizzare l'attenzione sulle procedure generali, anziché sui singoli elementi.

Inoltre, si dovrebbe tenere presente che la morfologia costruisce strutture non solo per la flessione di una parola, ma anche per derivazione di una parola dall'altra. Ad esempio, la parola in-ex-pugn-a-bilis contiene un tema, pugna, formato da una radice e una vocale tematica, due prefissi e due suffissi, secondo la struttura schematizzata nella figura, che riassume la catena derivativa da pugnare verbo $_{\rightarrow} \rightarrow$ con l'aggiunta dapprima del prefisso ex-: expugnare; $\rightarrow$ poi la formazione di un aggettivo con il suffisso -bilis: expugnabilis; $\rightarrow$ e infine l'aggiunta del prefisso negativo in-: inexpugnabilis. La struttura dei morfemi è indicata dal seguente diagramma ad albero: 


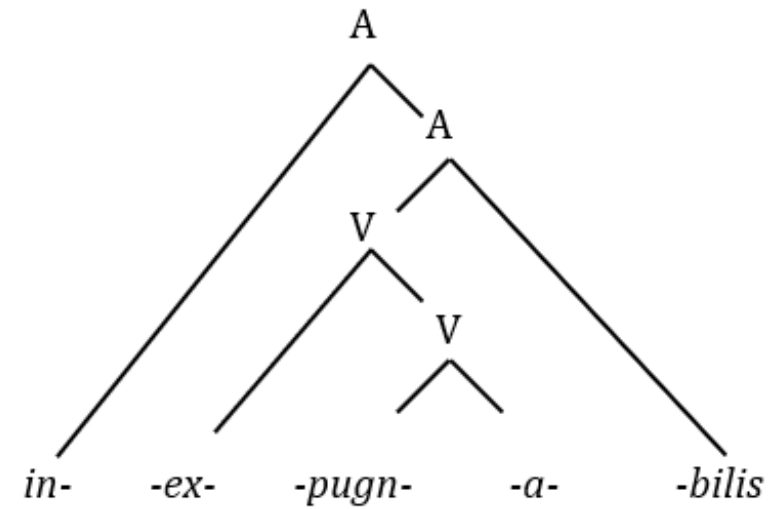

Il punto cruciale è che la struttura dei morfemi non è lineare, ma gerarchica, perché il prefisso in-, che è il primo nell'ordine lineare, è l'ultimo nell'ordine gerarchico.

\section{Problemi di sintassi}

Concludiamo con qualche considerazione su un punto fondamentale della sintassi: il concetto di sintagma. Come abbiamo visto nel passo di Prisciano citato nel paragrafo precedente, la grammatica antica conosceva solo le parole (dictiones) e le loro costruzioni per formare le frasi (orationes). Pertanto, le frasi erano concepite come sequenze lineari di parole, ciascuna classificata secondo il sistema delle parti del discorso. Fu il grande pedagogista boemo Comenio, alla metà del Seicento, a scoprire l'esistenza di unità intermedie tra la parola e la frase, introducendo il concetto di "gruppo di parole": in latino phrasis, da cui deriva l'inglese phrase, in italiano sintagma ${ }^{16}$.

L'analisi in sintagmi ci permette di capire che alcune parole formano un gruppo, mentre altre no: per mezzo dell'albero sintattico, è possibile visualizzare in modo preciso e nello stesso tempo intuitivo i rapporti tra le parole. L'individuazione dei sintagmi è un'operazione necessaria per la corretta comprensione delle frasi. Prendiamo ad esempio un celebre verso di Virgilio:

Ab love principium Musae Iovis omnia plena (Verg. Ecl. 3.60).

Da Giove il principio, o Muse, (oppure: "da Giove il principio della mia poesia”), tutto è pieno di Giove".

Ci sono due modi per analizzare la struttura del sintagma nominale a cui si riferisce la predicazione $a b$ love, da cui derivano due diversi significati (Cfr. l'analisi di PeLLACANI 2014). Il primo è analizzare il sintagma nominale principium Musae come formato da una testa e un complemento al genitivo, il secondo è invece analizzare il sintagma nominale come formato dalla sola testa principium, mentre Musae può 
essere inteso come un vocativo. Secondo le due diverse strutture sintagmatiche, la frase esprime due significati diversi: nella prima "da Giove il principio della Musa"; nella seconda "da Giove il principio, o Muse". Se la frase fosse una semplice sequenza lineare di parole, non riusciremmo a rendere conto della diversità di significato, che dipende appunto dal diverso raggruppamento delle parole in sintagmi.

La procedura fondamentale per la costruzione di un sintagma consiste nel combinare insieme più elementi per costruire strutture gerarchiche. L'operazione fondamentale è il raggruppamento di due parole all'interno di una categoria che le contiene entrambe. Chiamiamo X e Y le parti del discorso delle due parole che si combinano per formare il sintagma. Il costituente principale X è detto "testa" e il costituente subordinato Y è detto "complemento". L'intero sintagma prende il nome dalla propria testa, cioè SX è il sintagma di X:

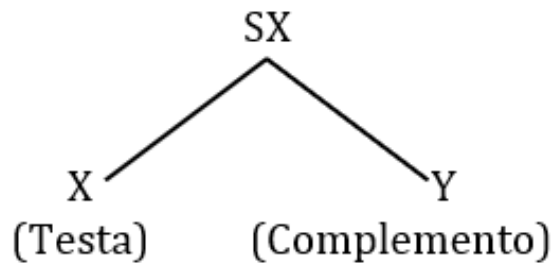

Se la testa è un verbo $(\mathrm{V})$ si forma un sintagma verbale (SV), se la testa è un nome (N) si forma un sintagma nominale (SN), e così via per il sintagma preposizionale (SP) e il sintagma aggettivale (SA).

La struttura dei sintagmi è universale, cioè si trova in tutte le lingue del mondo, latino compreso. Ad esempio, il sintagma nominale tribunus plebis "tribuno della plebe" ha il seguente albero sintattico:

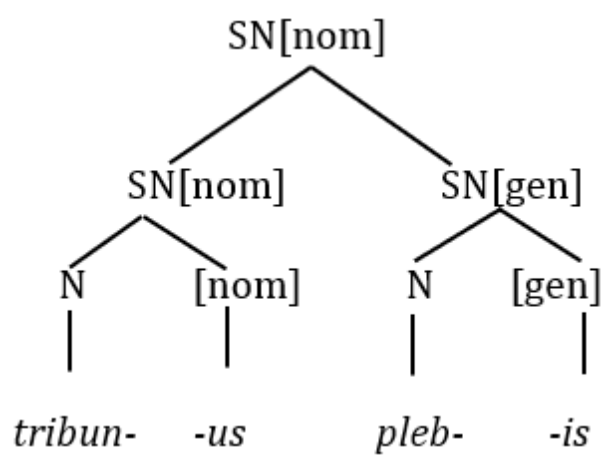

In questo sintagma troviamo due nomi: uno al nominativo tribunus e uno al genitivo plebis. Le parole latine hanno la struttura di sintagmi formati dal tema che rappresenta il nome astratto $(\mathrm{N})$ e dalla desinenza che introduce il caso. Le due parole si uniscono poi per formare il sintagma nominale tribunus plebis, dove la 
testa è tribunus e il complemento è plebis, parola che non può stare da sola, ma ha bisogno della testa a cui aggiungersi.

La principale differenza rispetto all'italiano sta nel fatto che il latino possiede le desinenze di caso, quindi il complemento è marcato dal caso genitivo. Si noti che il caso di plebis rimane fissato dal momento in cui la parola entra nel sintagma come complemento, mentre la testa tribūnus rimane libera di assumere il caso richiesto dalla frase, come il nominativo nel caso sia soggetto (ad es. tribunus plebis adest), l'accusativo nel caso sia oggetto (ad es. laudamus tribunum plebis), e così via.

Oltre alla testa, che è l'unico elemento obbligatorio, e al suo eventuale complemento, un sintagma può contenere anche altri elementi ${ }^{17}$. Un esempio è il sintagma nominale Helvetiorum iniuriae populi Romani "le offese degli Elvezi contro il popolo romano". Qui è presente un altro sintagma nominale al genitivo, Helvetiorum, chiamato "specificatore", che si colloca a sinistra sopra la testa iniuriae al nominativo, alla quale si accompagna il complemento al genitivo populi Romani, formato da un nome e un aggettivo. L'aggettivo è chiamato "aggiunto", per sottolineare il suo carattere facoltativo: a qualunque nome o sintagma nominale si può aggiungere un aggettivo: ad es. optimus tribunus militum, oppure antiquae Helvetiorum iniuriae populi Romani.

Per riassumere, un sintagma è formato sempre allo stesso modo. Ci deve essere per forza almeno una testa. La testa può prendere un complemento. Ci può essere poi uno specificatore, e infine a questa struttura di base si può aggiungere esternamente un numero indefinito di espansioni, cioè gli aggiunti, che sono tipicamente sintagmi aggettivali o preposizionali.

Notiamo infine che l'ordine delle parole nel sintagma nominale è più libero in latino rispetto ad una lingua come l'italiano, dove l'ordine è tendenzialmente fisso, cioè la testa precede il complemento: si può dire perciò lezione di latino, ma non *di latino lezione. Solo in poesia sono ammessi sintagmi del tipo la di lei paura (Carlo Porta). In latino, invece, le parole sono libere di muoversi: è ammesso sia l'ordine per cui la testa precede il complemento (liber Catulli), sia il contrario (Catulli liber), con lo stesso significato "il libro di Catullo".

Per spiegare questo fenomeno è possibile utilizzare il concetto intuitivo di "movimento sintattico". Partiamo dal sintagma nominale che già conosciamo, cioè quello formato dalla fusione della testa liber e del complemento Catulli:

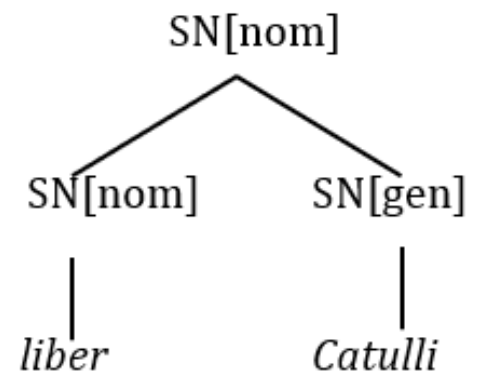


A partire da questa struttura, è possibile ipotizzare il movimento del complemento, che si sposta all'indietro, scavalcando la testa:

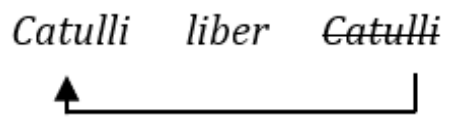

In tal modo si forma il sintagma Catulli liber, che ha lo stesso significato di liber Catulli, ma mette in maggior evidenza il nome di Catullo. Può essere interessante il confronto con l'inglese, dove il movimento non è ammesso nel genitivo preposizionale the book of Catullus, ma è obbligatorio nel genitivo sassone Catullus's book. Il concetto di movimento sintattico è dunque semplice, intuitivo, e di carattere universale. Si tratta di un'operazione molto semplice che noi tutti compiamo, quando scrivendo cancelliamo e spostiamo parole o gruppi di parole.

In sintesi, la struttura dei sintagmi si basa sui due semplici meccanismi: il raggruppamento e il movimento. L'ordine lineare degli elementi varia in base ai parametri delle singole lingue, ma la struttura dei sintagmi rimane la stessa in tutte le lingue. Quindi, il latino non è il caso isolato di una lingua strana, dove curiosamente l'ordine delle parole non conta. Anzi, per tradurre dal latino, bisogna in primo luogo riconoscere l'organizzazione dei sintagmi, così come essi si presentano nella concretezza di un testo, in cui l'ordine può essere stato perturbato da movimenti. Una delle cose che più colpisce in latino è la grande libertà di movimento, perché si tratta di testi letterari o addirittura poetici.

Una buona traduzione deve partire dall'analisi rigorosa delle strutture morfologiche e sintattiche presenti nel testo latino, ma deve poi essere affinata con sensibilità letteraria, per produrre un risultato che sia non solo grammaticalmente corretto, ma anche valido sotto il profilo stilistico ${ }^{18}$. La traduzione dal latino rimane perciò un insostituibile strumento educativo, che ci permette di far progredire da un lato la nostra capacità di analisi linguistica di un testo, dall'altro la nostra sensibilità letteraria, contribuendo alla creazione di un nostro stile personale, grazie al confronto con i grandi classici e al dialogo con la cultura antica ${ }^{19}$.

Quando gli studenti avranno risolto in maniera esatta il problema dell'analisi fonetica, morfologica e sintattica di una frase, la leggeranno con gli accenti giusti, e saranno in grado di capirne la struttura. Serviranno tempo ed esercizio per imparare anche a tradurre bene, perché la traduzione non è solo una scienza, ma anche un'arte, che richiede anni di esperienza per potersi affinare. Ma una volta divenuti padroni di un solido metodo di analisi grammaticale, difendibile sul piano scientifico, essi potranno esercitare un controllo razionale sulla materia che stanno apprendendo, e in tal modo acquisteranno sempre più fiducia nelle loro capacità, miglioreranno la conoscenza della loro madrelingua, e magari alla fine si appassioneranno al latino. 


\section{Bibliografia}

ANDREONI FonteCEDRo, E., Agosti, M. E SEnNI, C. (2017). Guida alla traduzione del testo latino. Roma.

BALBO, A. (2007). Insegnare latino. Sentieri di ricerca per una didattica ragionevole. Novara.

BALBO, A. (2017). Materiali e metodi per una didattica multimediale del latino. Bologna.

BAlbo, A. E Ricucci, M. (2014). Prospettive per l'insegnamento del latino. La didattica della lingua latina fra teoria e buone pratiche, Torino.

BALDACCI, M. (2012). Trattato di pedagogia generale. Roma.

BEnINCÀ, P. E PECA CONTI, R. (2003). Didattica delle lingue classiche e linguistica teorica. Università e Scuola, 8(2), 38-53.

BERCHTOLD, V., JANKA, M. \& SCHAUER, M. (Hrsg.) (2016). Adeamus! München.

BETTINI, M. (2012). Vertere: un'antropologia della traduzione nella cultura antica. Torino.

BETTINI, M. E SHORT, W. (a cura di) (2014). Con i Romani: un'antropologia della cultura antica. Bologna.

CARDinale, U. (a cura di) (2008). Nuove chiavi per insegnare il classico. Torino.

CARDINALETTI, A. (2006). Una proposta per l'insegnamento linguistico del latino e del greco antico: alcune considerazioni generali. In U. CARDINALE (a cura di), Essere e divenire del "classico" (pp. 330-337). Torino.

CHOMSKY, N \& HALLE, M. (1968). The Sound Pattern of English. New York.

Collins, A., Brown, J.S. E NeWman, S.E. (1995). L'apprendistato cognitivo, trad. it. in C. Pontecorvo, A. Ajello \& C. Zucchermaglio (a cura di), I contesti sociali dell'apprendimento (pp. 181-231). Milano.

Colombo, A. \& GRAFFI, G. (2017). Capire la grammatica. Il contributo della linguistica. Roma.

DEWEY, J. (1961). Come pensiamo. Una riformulazione del rapporto tra il pensiero riflessivo e l'educazione, trad. it. Firenze, (ed. orig. 1933).

EMBICK, D. (2015). The Morpheme: A Theoretical Introduction. Berlin.

GARBugino, G. (1993). Latino ed educazione linguistica. Padova.

GIORDANO RAMPIONI, A. (2010). Manuale per l'insegnamento del latino. Bologna.

GIOSEFFI, M. (a cura di) (2010). Uso, riuso e abuso dei testi classici. Milano.

GIUSTI, G. E ONIGA, R. (2006). La struttura del sintagma nominale latino. In R. Oniga E L. Zennaro (a cura di), Atti della Giornata di linguistica latina (pp. 71-100). Venezia.

HAEGEMAN, L. (1997). The New Comparative Syntax. London.

HOFMANN, J.B. E SZANTYR, A. (2002). Stilistica latina (trad. it. a cura di A. Traina). Bologna.

IOVINO, R. (2013). Come la linguistica teorica può contribuire a rinnovare l'insegnamento della sintassi nominale latina. Lingue Antiche e Moderne, 2, 5-31. 
LARSON, R. (2010). Grammar as Science. Cambridge (Mass.).

LISTER, B. (2012). I classici in Inghilterra: l'insegnamento delle lingue nel loro contesto culturale. In L. Canfora \& U. Cardinale (a cura di), Disegnare il futuro con intelligenza antica (pp. 301-315). Bologna.

MALASPINA, E. (2013). Insegnare oggi latino e greco nei licei. In Pagnotta, F. (a cura di), L'età di internet. Umanità, cultura, educazione (pp. 37-52). Firenze.

MARINO, R. (2006). La traduzione: un dialogo fra due culture. In U. Cardinale (a cura di), Essere e divenire del classico (pp. 357-367). Torino.

MAROTTA, G. (2006). L'algoritmo accentuale latino nel confronto di due teorie fonologiche. In R. Oniga E L. Zennaro (a cura di), Atti della Giornata di linguistica latina (pp. 133158). Venezia.

MARTINI, B. (2005). Formare ai saperi. Per una pedagogia della conoscenza. Milano.

MESTER, A. (1994). The Quantitative Trochee in Latin. Natural Language and Linguistic Theory, 12, 1-61.

MIRAGLIA, L. (2004). Metodo natura e storia culturale. In G. Milanese (a cura di), A ciascuno il suo latino: la didattica delle lingue classiche dalla scuola di base all'università (pp.23-62). Galatina.

Most, W.G. (1958). Latin by the Natural Method. Chicago.

ONIGA, R. (1998). Teorie linguistiche e didattica del latino”. In B. García-Hernández (Ed.), Estudios de lingüística latina (pp. 613-626).

ONIGA, R. (2007). Il latino. Breve introduzione linguistica. Nuova edizione riveduta e ampliata Milano (2004 ${ }^{1}$ ); trad. ingl. Latin: A Linguistic Introduction, Oxford 2014.

ONIGA, R. (2010). I fondamenti linguistici della metrica latina arcaica. Lexis, 28, 343-368.

ONIGA, R. (2012). Insegnare latino con il metodo neo-comparativo. In R. Oniga \& U. Cardinale (a cura di), Lingue antiche e moderne dai licei alle università (pp. 101-121). Bologna.

ONIGA, R. (2017). La storia della parola phrasis dall'antichità ad oggi. In S. Rocca (a cura di), Latina didaxis XXXI (Atti del Convegno di Genova 17 maggio 2016, pp. 29-57). Milano.

ONIGA, R. (2018). Il latino nella formazione intellettuale europea in età moderna e contemporanea. Paideia, 73, 593-612.

ONIGA, R. (2020). Riscoprire la grammatica. Il metodo neo-comparativo per l'apprendimento del latino. Udine.

ONIGA, R., IOVINO, R. E GIUSTI, G. (Eds.) (2011). Formal Linguistics and the Teaching of Latin. Newcastle upos Tyne $\mathcal{E}$ Cambridge Scholars Publishing.

OTERO, C.P. (2003). Chomsky on Democracy and Education. London E New York.

ØRBERG, H.H. (1954). Lingua latina secundum naturae rationem explicata, København, The Nature Method Language Institutes; ed. it. Lingua latina per se illustrata, Montella, Accademia Vivarium Novum, 1997. 
PellacANI, D. (2014). Virgilio ecl. 3, 60: ambiguità sintattica e arte allusiva. Paideia, 69, 457-466.

Penello, N. (2006). Applicazioni di elementi di linguistica formale alla didattica del latino. In R. Oniga \& L. Zennaro (a cura di), Atti della Giornata di linguistica latina (pp. 159178). Venezia, Cafoscarina.

PeZziNI, G. E TAYLOR, B. (Eds.) (2019). Language and Nature in the Classical Roman World. Cambridge.

PIERI, M.P. (2005). La didattica del latino. Roma.

Puliga, D. (2003). Percorsi della cultura latina. Per una didattica sostenibile. Roma.

RICUCCI, M. (2013). Per un apprendimento linguistico secondo il metodo neo-comparativo: note storico-concettuali. Lingue Antiche e Moderne, 2, 55-78.

RoccA, S. (a cura di). (2006). Latina Didaxis XXI, Dalla disciplina alla didattica, Genova.

Traina, A. E Bernardi PerinI, G. (1992). Propedeutica al latino universitario (Quarta edizione a cura di C. Marangoni). Bologna.

VALENTI, R. (2011). Il latino dentro e oltre la scuola. Memoria, identità, futuro. Napoli.

\section{Notas}

${ }^{1}$ Sui pregi e i limiti degli strumenti multimediali per il latino, cfr. BALBO (2017).

2 Per una panoramica aggiornata sulla didattica del latino, cfr. PIERI (2005); RocCA (2006); BALBO (2007); CARDINALE (2008); GIORDANO RAMPIONI (2010); VALENTI (2011); MALASPINA (2013); BALBO \& RICUCCI (2014); ONIGA (2020).

${ }^{3}$ Cfr. ad es. il panorama storico in GIOSEFFI (2010); ONIGA (2018).

${ }^{4}$ Sull'antropologia del mondo antico, cfr. in particolare BETTINI (2012), con riferimento al problema della traduzione, e inoltre la raccolta di saggi a cura di BETTINI \& SHORT (2014); applicazioni didattiche in PULIGA (2003).

${ }^{5}$ La genesi e la struttura di questo corso è descritta da LISTER (2012). Un'impostazione simile si ritrova nel manuale a cura di BERCHTOLD et al. (2016).

${ }^{6}$ Si veda in proposito il recente volume curato da PEZZINI \& TAYLOR (2019).

7 HaEgeman (1997); ONIGA (1998); ONIGA \& IOVINo \& Giusti (2011); ONIGA (2012; 2020); IOVINO (2013); RICUCCI (2013).

${ }^{8}$ Benincà E Peca Conti (2003); Cardinaletti (2006); Penello (2006); Oniga (2007); COLOMBO \& GRAFFI (2017).

${ }^{9}$ LARSON (2010).

10 Il tema è stato proposto e sviluppato in GARBUGINO (1993).

${ }^{11}$ Sui rapporti tra CHOMSKY e DEWEY, cfr. ad es., OTERO (2003).

${ }_{12}$ Per una introduzione, cfr. MARTINI (2005); BALDACCI (2012). 
${ }^{13}$ Per una esposizione sistematica, cfr. ONIGA (2020).

${ }^{14}$ Per una descrizione dettagliata dell'algoritmo accentuale latino, cfr. MAROTTA (2006) e ONIGA (2010).

${ }^{15}$ Gli unici monosillabi brevi sono gli elementi enclitici, privi di accento proprio, come -que, di cui parleremo nel prossimo paragrafo.

${ }^{16}$ La storia del concetto è presentata in ONIGA (2017).

${ }_{17}$ Per un'analisi dettagliata della struttura del sintagma nominale in latino, cfr. GIUSTI $E$ ONIGA (2006).

${ }^{18}$ Per una introduzione alla stilistica latina, cfr. HOFMANN E SZANTYR (2002).

${ }_{19}$ Importanti osservazioni su questo aspetto imprescindibile nella didattica del latino si trovano in MARINO (2006); ANDREONI FONTECEDRO et al. (2017). 